\title{
One dimensional modeling of the thermoelastic behavior of shape memory alloys
}

\author{
Belkacem Meddour $^{1, a}$, Hamma Zedira ${ }^{2}$ And Hamid Djebaili ${ }^{2}$ \\ 1 University Hadj Lakhdar, Batna, Algeria \\ 2 Labo LaSPI2A, University Abbas Laghrour, Departement of sciences and technology, Khenchela, Algeria
}

Received 17 December 2013, Accepted 20 May 2014

\begin{abstract}
Many facets of the behavior of shape memory alloys have enormously attracted scientists and researchers, studying and modeling their behavior are still problematic. It is in this context that several models have been developed using a lot of approaches ranging from microscopic to macroscopic scale. In this paper we have considered the superthermal effect (thermoelastic) of shape memory alloys and we have adopted a macroscopic approach. The model takes in account only macroscopic observations, it was built basing on thermodynamic laws of thermodynamics and simple assumptions in order to simplify as much as possible its use in engineering without losing accuracy. To validate the model, we have simulated numerically the response and compared it to experimental data.
\end{abstract}

Key words: Stress / thermoelastic behavior / simulation / transformation / thermomechanics

\section{Introduction}

During these last decades, materials have seen major development and application in different areas; among this category of materials we distinguish shape memory alloys (SMAs), which are known by the property to recover their initial shape by simple heating after having been deformed under an applied mechanical load, it is the simple shape memory effect. Other properties can be exhibited by these materials after combining mechanical and thermal loads and include: double shape memory effect, superelasticity (pseudoelasticity) [1,2], superthermal effect (thermoelastic) [3], damping effect [4].

These properties derive from phases transformations i.e. higher temperature phase (austenite) to lower temperature phase (martensite).

It is important to observe that these phases transformations do not occur with diffusion but rather with displacement i.e. displacement at a distance less than interatomic $[5,6]$.

Previous cited properties are at the origin of various applications of SMAs, used in many varied fields: automotive industry, fire watch devices, aeronautics, medical devices [7].

To understand the thermomechanical behavior of these materials and to use it in engineering, various models have been developed; they can be classified into

\footnotetext{
${ }^{a}$ Corresponding author: samsum66@gmail.com
}

great categories: micromechanical and macroscopic ones. Most of the micromechanical models for polycrystalline SMAs are based on a self consistent type of averaging methods. Let us mention some models as micromechanical approaches for modeling pseudoelasticity [8,9] developed model for polycrystalline SMA [10], micromechanical model for multivariant SMA [11].

The macroscopic models seem less accurate than previous models but are used in engineering. We can include the Tanaka's model to describe pseudoelasticity and simple memory effect [12]; the macroscopic model developed by Berveiller et al. [13]; the one-dimensional model of SMA developed by Brinson et al. [14]; the phenomenological model to describe the two way shape memory effect of shape SMA [15]; the improvement to SMAs models carried out by Raniecki and Lexcellent [16]; the unified constitutive model developed by Lagoudas [17]; the extended model 3D developed by Lagoudas and Entchev to consider the stress-induced transformation by allowing both transformation and plastic strains as a result of the applied load [18].

This paper is intended to develop a macroscopic constitutive model to describe the isobaric thermal cycle of the SMA using thermodynamics laws, and constitutive equations.

The first section is devoted to write expression of the potential and constitutive equations, the last one is consecrated to the application and validation of the model. 


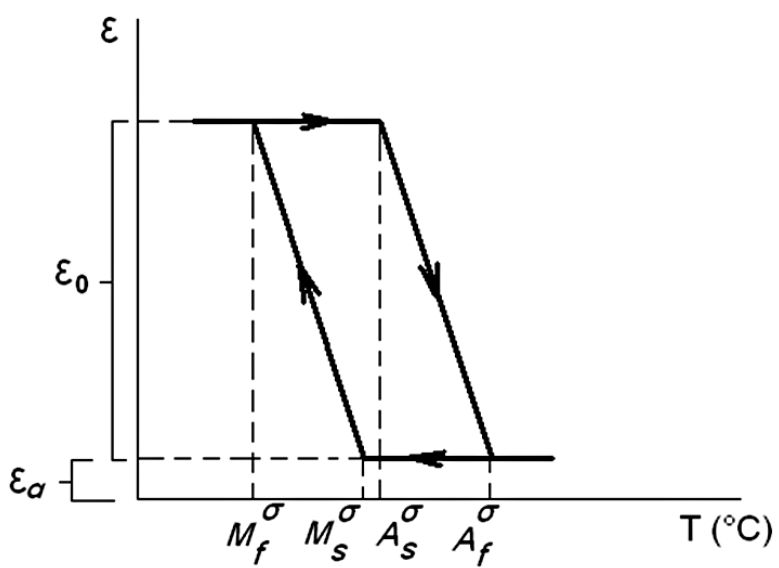

Fig. 1. Superthermal effect (under $\sigma=\sigma_{0}$ ).

$\varepsilon_{0}$ : Maximum deformation of transformation.

$\varepsilon_{a}$ : Deformation before transformation.

$M_{s}^{\sigma}$ : Temperature of transformation. start $\mathrm{A} \rightarrow \mathrm{M}$ under stress $\sigma$.

$M_{f}^{\sigma}$ : Temperature of transformation finish $\mathrm{A} \rightarrow \mathrm{M}$ under stress $\sigma$.

$A_{s}^{\sigma}$ : Temperature of transformation start $\mathrm{M} \rightarrow \mathrm{A}$ under stress $\sigma$

$A_{f}^{\sigma}$ : Temperature of transformation finish $\mathrm{M} \rightarrow \mathrm{A}$ under stress $\sigma$.

\section{Methods}

\subsection{Presentation of the subject}

Superthermal effect is obtained with applying a thermomechanical loading cycle. Under a constant stress $(\sigma=$ $\left.\sigma_{0}\right)$ and cooling below $M_{f}^{\sigma}$ then the material undergoes a large deformation, it is because of transformation of austenite to martensite, that was the direct transformation $\mathrm{A} \rightarrow \mathrm{M}$. When heating above $A_{f}^{\sigma}$ the previous deformation is recovered because of transformation of martensite to austenite, it is the reverse transformation $\mathrm{M} \rightarrow \mathrm{A}$ (Fig. 1).

\subsection{Expression of thermodynamic potential}

We consider a representative elementary volume REV of austenite with sufficient size to have a fraction of martensite when the transformation occurs (Fig. 3):

fraction of martensite: $f=\frac{V_{M}}{V}$

The macroscopic strain is written as:

$$
E=E^{e}+\varepsilon^{t}
$$

- $\varepsilon^{t}$ : deformation due to austenite transformation to martensite directly related to the formation of Martensite:

$$
\varepsilon^{t}=f \varepsilon_{0}
$$

- $E^{e}$ : overall elastic deformation:

$$
E^{e}=f \varepsilon_{M}^{e}+(1-f) \varepsilon_{A}^{e}
$$

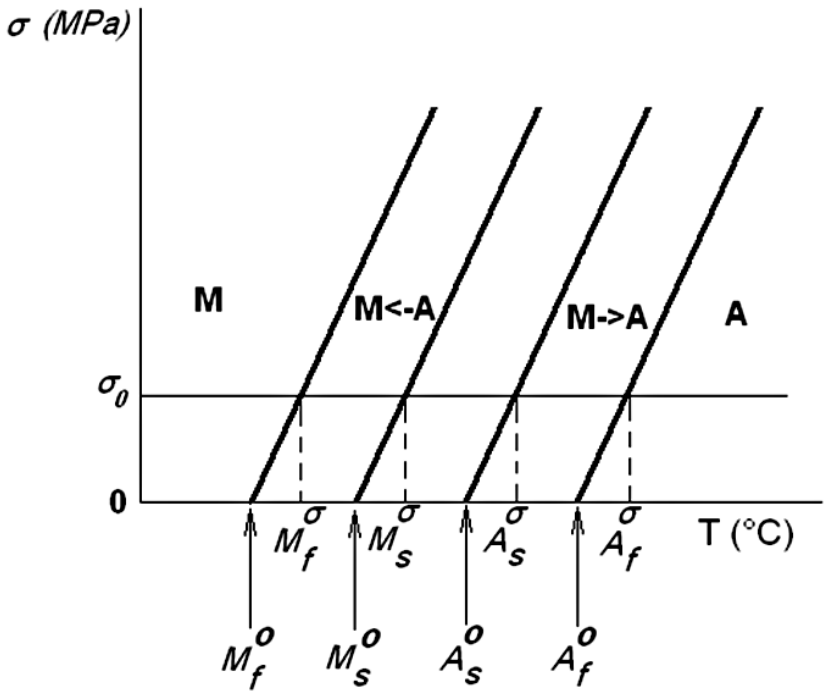

Fig. 2. Phase diagram of SMA.

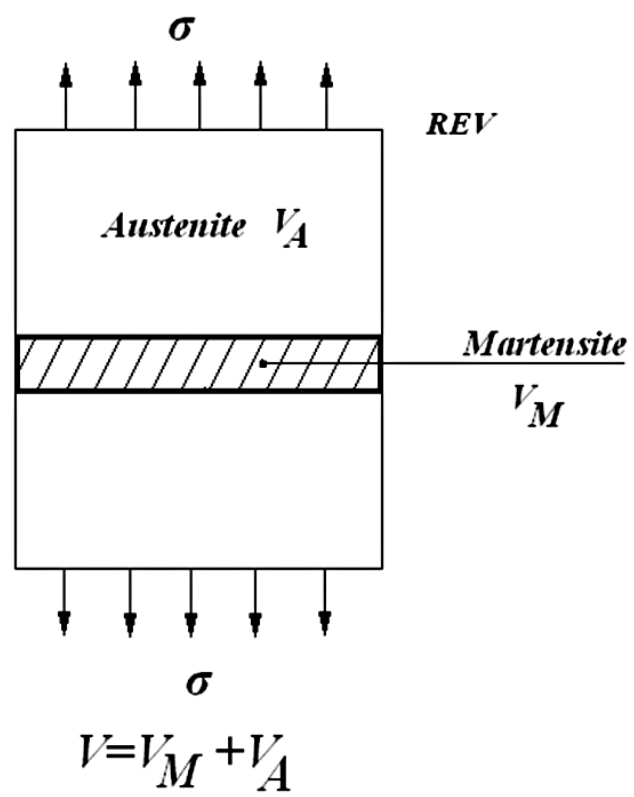

Fig. 3. Representative elementary volume.

- $\varepsilon_{M}^{e} ; \varepsilon_{A}^{e}$ : respectively elastic deformation of formed martensite and elastic deformation of remaining austenite:

$$
\varepsilon_{M}^{e}=\frac{\sigma}{E_{M}} ; \quad \varepsilon_{M}^{e}=\frac{\sigma}{E_{A}}
$$

- $E_{M} ; E_{A}$ : respectively Young modulus of martensite and austenite.

The thermodynamic potential is Gibbs free energy:

$$
G(\sigma, T, f)=W_{m}+W_{c h}+W_{i n t}
$$

- $W_{m}$ : mechanical energy:

$$
W_{m}=-\sigma E
$$


Using equations (1)-(4) $W_{m}$ will be written:

$$
W_{m}=-\frac{\sigma^{2}}{2}\left(\frac{1-f}{E_{A}}+\frac{f}{E_{M}}\right)-\sigma f \varepsilon_{0}
$$

- $W_{c h}$ : chemical free energy due to phase change;

$$
W_{c h}=f B\left(T-T_{0}\right)
$$

$B$ : proportional coefficient to be identified by test $[\mathrm{MPa} / \mathrm{K}]$.

$T_{0}$ : reference temperature; $T_{0}=M_{s}^{0}\left(M_{s}^{0}\right.$ temperature of transformation start of martensite) $[\mathrm{K}]$.

- $W_{\text {int }}$ : interaction energy; according to many authors this energy is a function of the fraction of martensite $[19,20]$.

We can choose the expression:

$$
W_{i n t}=C f(f-1)
$$

$C$ : proportional coefficient to be identified by test [MPa]. Consequently, Gibbs free energy can be written:

$$
\begin{aligned}
G(\sigma, T, f)=- & \frac{\sigma^{2}}{2}\left(\frac{1-f}{E_{A}}+\frac{f}{E_{M}}\right) \\
& -\sigma f \varepsilon_{0}+f B\left(T-T_{0}\right)+C f(f-1)
\end{aligned}
$$

$E_{A} ; E_{M}$ : Young modulus of austenite and martensite respectively.

Driving force $F^{t h}$ is derived from the potential $G(\sigma, T, f)$.

Because of dissipation due to martensite transformation, Clausius inequality gives:

$$
-\frac{\partial G}{\partial f} \frac{\mathrm{d} f}{\mathrm{~d} t} \geq 0
$$

Let us write

$$
\begin{gathered}
F^{t h}=-\frac{\partial G}{\partial f} \\
F^{t h}=\frac{\sigma^{2}}{2}\left(\frac{1}{E_{M}}-\frac{1}{E_{A}}\right)+\sigma \varepsilon_{0}-B\left(T-T_{0}\right)-C(2 f-1)
\end{gathered}
$$

It should be important to observe the hysteresis appearing when the inverse transformation happens, dissipation takes place. The dissipative force $F^{d i}$ is opposed to $F^{t h}$ :

$$
F^{t h}=F^{d i}
$$

$F^{d i}$ can be given as following:

$F^{d i}=K f+H ; K$ and $H$ are constants to be determined.

When considering the direct and reverse transformations, we can deduce:

$$
F^{t h}= \begin{cases}F^{d i} ; \frac{\mathrm{d} f}{\mathrm{~d} t}>0 & \text { Forward transformation } \\ -F^{d i} ; \frac{\mathrm{d} f}{\mathrm{~d} t}<0 & \text { Reverse transformation }\end{cases}
$$

\subsection{Constitutive equations}

\subsubsection{Forward transformation}

From Equation (13) we obtain:

$$
F^{t h}-F^{d i}=0
$$

Using consistency conditions we can write:

$$
\dot{F}^{t h}-\dot{F^{d}} d i=0
$$

Finally, we deduce:

$$
\begin{array}{r}
\frac{\sigma^{2}}{2}\left(\frac{1}{E_{M}}-\frac{1}{E_{A}}\right)+\sigma \varepsilon_{0}-B\left(T-T_{0}\right)-C(2 f-1) \\
-K f-H=0
\end{array}
$$

Let us write:

$$
\begin{array}{r}
\frac{\sigma^{2}}{2}\left(\frac{1}{E_{M}}-\frac{1}{E_{A}}\right)+\sigma \varepsilon_{0}-B\left(T-T_{0}\right)-C(2 f-1) \\
-K f-H=\varphi^{1}(\sigma, T, f) \\
\frac{\mathrm{d} \varphi(\sigma, T, f)}{\mathrm{d} t}=\frac{\partial \varphi}{\partial \sigma} \frac{\mathrm{d} \sigma}{\mathrm{d} t}+\frac{\partial \varphi}{\partial T} \frac{\mathrm{d} T}{\mathrm{~d} t}+\frac{\partial \varphi}{\partial f} \frac{\mathrm{d} f}{\mathrm{~d} t}=0
\end{array}
$$

Using (18) and (19); finally we obtain:

$$
\left[\sigma\left(\frac{1}{E_{M}}-\frac{1}{E_{A}}\right)+\varepsilon_{0}\right] \dot{\sigma}-B \dot{T}-(2 C+K) \dot{f}=0 ; \quad \dot{f}>0
$$

Equation (11) determines the evolution of the transformation.

In this case $\dot{\sigma}=0$, then (20) can be written:

$$
\dot{f}=-\frac{B \dot{T}}{2 C+K} ;(\dot{f}>0)(\dot{T}<0)
$$

\subsubsection{Reverse transformation}

Using the same procedure as previously, we obtain:

$$
\begin{array}{r}
\frac{\sigma^{2}}{2}\left(\frac{1}{E_{M}}-\frac{1}{E_{A}}\right)+\sigma \varepsilon_{0}-B\left(T-T_{0}\right)-C(2 f-1) \\
+K f+H=0
\end{array}
$$

$$
\begin{array}{r}
\frac{\sigma^{2}}{2}\left(\frac{1}{E_{M}}-\frac{1}{E_{A}}\right)+\sigma \varepsilon_{0}-B\left(T-T_{0}\right)-C(2 f-1) \\
+K f+H=\varphi^{2}(\sigma, T, f)
\end{array}
$$

$$
\left[\sigma\left(\frac{1}{E_{M}}-\frac{1}{E_{A}}\right)-\varepsilon_{0}\right] \sigma \dot{-} B T-(2 \dot{C}-K) \dot{f}=0 ; \dot{f}<0
$$

$$
\dot{f}=-\frac{B \dot{T}}{2 C-K} ; \quad(\dot{f}<0) ; \quad(\dot{T}>0)
$$




\subsection{Determination of coefficients $B, C, K, H$}

\subsubsection{Forward transformation}

We use:

$$
\begin{aligned}
& \varphi^{1}\left(\sigma=\sigma_{0}, T, f\right)=0 ; \quad T=M_{s}^{\sigma} ; \quad f=0 \\
& \varphi^{1}\left(\sigma=\sigma_{0}, T, f\right)=0 ; \quad T=M_{f}^{\sigma} ; \quad f=1
\end{aligned}
$$

\subsubsection{Reverse transformation}

We use:

$$
\begin{aligned}
& \varphi^{2}\left(\sigma=\sigma_{0}, T, f\right)=0 ; \quad T=A_{s}^{\sigma} ; \quad f=1 \\
& \varphi^{2}\left(\sigma=\sigma_{0}, T, f\right)=0 ; \quad T=A_{f}^{\sigma} ; \quad f=0
\end{aligned}
$$

Resolving the system of equations we obtain:

$$
\begin{aligned}
B=\left[2 \sigma_{0}^{2}\left(\frac{1}{E_{M}}-\frac{1}{E_{A}}\right)+4 \sigma_{0} \varepsilon_{0}\right] / & \\
& \left(M_{s}^{\sigma}+M_{f}^{\sigma}+A_{s}^{\sigma}+A_{f}^{\sigma}\right)
\end{aligned}
$$

$C=\sigma_{0}^{2}\left(\frac{1}{E_{M}}-\frac{1}{E_{A}}\right)+2 \sigma_{0} \varepsilon_{0}-\frac{B\left(M_{f}^{\sigma}+A_{s}^{\sigma}-2 M_{s}^{0}\right)}{2}$

$K=\frac{\sigma_{0}^{2}}{2}\left(\frac{1}{E_{M}}-\frac{1}{E_{A}}\right)+\sigma_{0} \varepsilon_{0}-B\left(M_{s}^{\sigma}-M_{s}^{0}\right)+C$

$H=\frac{\sigma_{0}^{2}}{2}\left(\frac{1}{E_{M}}-\frac{1}{E_{A}}\right)+\sigma_{0} \varepsilon_{0}-B\left(M_{f}^{\sigma}-M_{s}^{0}\right)-C-K$

\subsection{Model parameters and application}

The parameters of the model were determined from a test which was performed on CuZnAl specimen (Fig. 4), and are presented in Table 1.

\section{Results and discussion}

We wrote the constitutive equations and conditions of direct transformations (austenite to martensite) and reverse (martensite to austenite) in the assumption that the cycle is as described in Figure 1.

Experimental data were provided from (Bourbon et al. [18]) work. The test performed on the alloy CuZnAl [18], under applied stress $\sigma_{0}=65 \mathrm{MPa}$.

To simulate the response of the model we have used an algorithm and we have obtained the results given in Figures 4 and 5 .

By analyzing results of each of previous figures (Figs. 4 and 5) we observe a hysteresis; this one is due to dissipating phenomena.

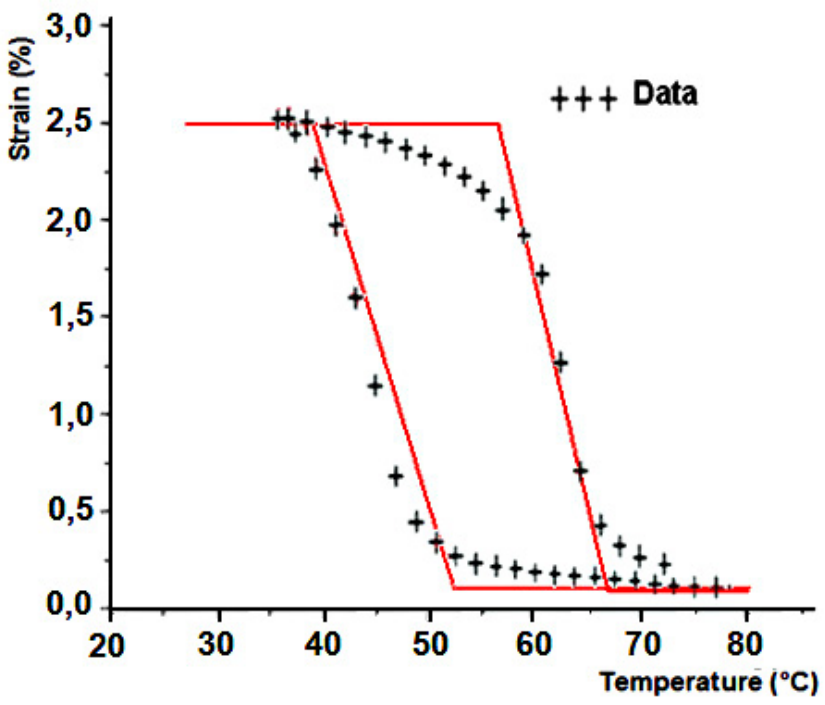

Fig. 4. Results of simulation $\left(\sigma_{0}=65 \mathrm{MPa}\right)$ [18].

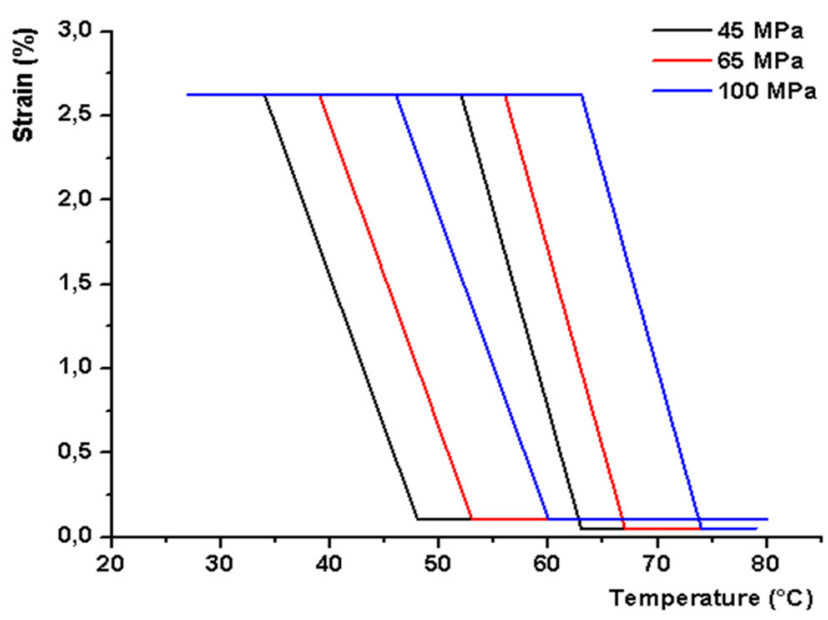

Fig. 5. Response of the model $\left(\sigma_{0}=45 \mathrm{MPa} ; \sigma_{0}=65 \mathrm{MPa}\right.$; $\left.\sigma_{0}=100 \mathrm{MPa}\right)$.

The results of simulation are purely linear. In Figure 4 we see that simulated results are in agreement with experimental data despite the offsets which could be observable. An offset of deformation at the corner of the hysteresis could be noticed $(\Delta \varepsilon \leq 0.001)$ approximately $3 \%$. In the bottom we observe a slight offset after the reverse transformation which is approximately 0.0005 approximately $2 \%$.

Figure 5 shows an anticipated response in terms of temperatures offset and maximum deformations.

\section{Conclusions}

Despite the variety of models proposed to describe the thermomechanical behavior of shape memory materials, only the cost and precision can differentiate them from each other. Our model was built starting from simple assumptions and using a simple formalism. 
Table 1. Parameters of the model $[18,20] . B=1.149001 \mathrm{E}-01 \mathrm{MPa} ; C=6.755679 \mathrm{E}-01 \mathrm{MPa} ; K=1.762350 \mathrm{E}-01 \mathrm{MPa}$; $H=9.390822 \mathrm{E}-01 \mathrm{MPa}$.

\begin{tabular}{cccccc}
\hline Parameters & Values & Parameters & Values & Parameters & Values \\
\hline$M_{s}^{0}(\mathrm{~K})$ & 313 & $M_{s}^{\sigma}(\mathrm{K})$ & 324 & $E_{A}(\mathrm{MPa})$ & 72000 \\
$M_{f}^{0}(\mathrm{~K})$ & 303 & $M_{f}^{\sigma}(\mathrm{K})$ & 311 & $E_{M}(\mathrm{MPa})$ & 70000 \\
$A_{s}^{0}(\mathrm{~K})$ & 315 & $A_{s}^{\sigma}(\mathrm{K})$ & 330 & $\varepsilon_{0}$ & 0.023937 \\
$A_{f}^{0}(\mathrm{~K})$ & 325 & $A_{f}^{\sigma}(\mathrm{K})$ & 340 & $\varepsilon_{a}$ & 0.001063 \\
\hline
\end{tabular}

We compared the response to the experimental results, and we conclude that:

1. The simulation results are generally in agreement with the experimental data especially under $\sigma_{0}=65 \mathrm{MPa}$.

2. The cycle shows a complete loop i.e. the specimen recovers its initial state.

3. This model can be used in engineering.

The previous constitutive model developed with a phenomenological approach was built using a simple formalism. This model was able to predict the response under a thermomechanical load. The obtained results by numerical simulation of the isobaric cyclic behavior are mostly consistent with experimental data and with acceptable accuracy. Finally we think that it can be used in engineering fields.

\section{References}

[1] S. Miyazaki, Y. Ohmi, K. Otsuka, Y. Suzuki, Characteristics of deformation and transformation pseudoelasticity in Ti-Ni alloys, J. Phys. 43 (1982) 255-260

[2] S.S. Miyazaki, T. Imai, Y. Igo, K. Otsuka, Effect of cyclic deformation on the pseudoelasticity characteristics of TiNi alloys, Metall. Trans. A 17 (1986) 115-120

[3] G. Bourbon, C. Lexcellent, S. Leclercq, Modelling of the non-isothermal cyclic behaviour of a polycrystalline $\mathrm{Cu}-$ Zn-Al shape memory alloy, J. Phys. IV 5 (1995) 221-226

[4] H.C. Lin, S.K. Wu, M.T. Yeh, Metall. Trans. A 24 (1993) 2189

[5] K. Otsuka, Perspective of research on martensitic transformations present and future, J. Phys. IV 11 (2001) 3-9

[6] J. Van Humbeeck, La transformation martensitique, dans Technologie des Alliages à Mémoire de Forme, Hermes 1993, Chap 3, pp. 63-88

[7] T. Duerig, A. Pelton, D. Stockel, An overview of nitinol medical applications, Mater. Sci. Eng. 273-275 (1999) 149-160

[8] E. Patoor, A. Eberhardt, M. Berveiller, Potentiel pseudoélastique et plasticité de transformation martensitique dans les mono et polycristaux metalliques, Acta Metall. 35 (1987) 2779-2789
[9] E. Patoor, A. Eberhardt, M. Berveiller, Micromechanical modelling of superelasticity in shape memory alloys, J. Phys. IV 6 (1996) 277-292

[10] D.C. Lagoudas, A. Bhattacharyya, On the Correspondence between Micromechanical Models for Shape Memory Alloys and the Preisach Model for Hysteresis, Math. Mech. Solids 2 (1997) 405-440

[11] X. Gao, L.C. Brinson, A simplified multivariant SMA model based on invariant plane nature of martensitic transformation, J. Intell. Mater. Syst. Struct. 13 (2002) $795-810$

[12] K. Tanaka, S. Kobayashi, Y. Sato, Thermomechanics of transformation pseudoelasticity and shape memory effect in alloys, Int. J. Plasticity 2 (1986) 59-72

[13] M. Berveiller, E. Patoor, M. Buisson, Thermomechanical constitutive equations for shape memory alloys, J. Phys. IV 1, C.4,387, European Symposium on Martensitic Transformation and Shape Memory Properties, 1991

[14] L.C. Brinson, One-dimensional constitutive behavior of shape memory alloys: Thermomechanical derivation with non-constant material functions and redefined martensite internal variable, J. Intell. Mater. Syst. Struct. 4 (1993) 229-242

[15] C. Lexcellent, S. Leclerq, B. Gabry, G. Bourbon, The two way shape memory effect of shape memory alloys: an experimental study and a phenomenological model, Int. J. Plasticity 16 (2000) 1155-1168

[16] B. Raniecki, C. Lexcellent, RL-models of pseudoelasticity and their specification for some shape memory solids, Eur. J. Mech. A/Solids 13 (1994) 21-50

[17] D.C. Lagoudas, Z. Bo, M.A. Qidwai, A unified thermodynamic constitutive model for SMA and finite element analysis of active metal matrix composites, Mech. Compos. Mater. Struct. 3 (1996) 153-179

[18] D.C. Lagoudas, P.B. Entchev, Modeling of transformation-induced plasticity and its effect on the behavior of porous shape memory alloys, Part I: Constitutive model for fully dense SMAs, Mech. Mater. 36 (2004) 865-892

[19] B. Raniecki, C. Lexcellent, Thermodynamics of isotropic pseudoelasticity in shape memory alloys, Eur. J. Mech. A/Solids 17 (1998) 185-205

[20] Shape Memory Applications Inc. http://www.sma-inc. com 